\title{
New Paradigms in Mediation: Thinking About Our Thinking
}

\section{Larry S Fong}

Trainers of mediators are often asked questions about how to deal with clients who are very protracted in their conflict. Some of the following thoughts, gleaned from preconference institutes conducted by the author at the last three Academy of Family Mediator conferences, may provide the mediator with suggestions leading to more successful mediations. Success is an attitude on the mediator's part: success is aided when a mediator thinks about how she or he thinks.

- A paradigm can be described as the rules of the game, the way things are, parameters, or boundaries and how you navigate within these boundaries. Marriage is a good example of a paradigm: it has its own set of rules, and these rules assist the members of the couple to understand how to deal with one another. Another way to describe a paradigm is to say that it is the way we perceive our surroundings.

- A paradigm shift occurs when the "rules of the game" change a person's life. For example, a paradigm shift occurs when separation or divorce takes place. Those who best understand and anticipate paradigm shifts will deal best with the future.

- Paradigms make us comfortable, for a familiar paradigm enables us to have a certain degree of confidence in outcomes. However, no paradigm can last forever or will always hold true. One paradigm that has recently shifted, for example, is that "marriage is forever".

- When things change significantly, we call this a paradigm shift. To make significant changes, individuals need to alter the way they view the world and how they resolve difficulties. A paradigm is really a set way of thinking about the world. With the changing of roles, paradigms also change. Thus, changing a person's role can cause a paradigm shift.

- People tend to prefer the status quo and functioning within familiar paradigms. Thus, they resist changing paradigms or changing themselves.

- Families who interact with one another involve themselves in acknowledged and unacknowledged family "games". They act according to specific sets of rules and tacit understandings that are intricately and inextricably entwined. These family games can be either functional or dysfunctional.

- In these games, family members try to unilaterally control each other's behaviours. Dysfunctional families can be explained as those in which family members utilise inappropriate control mechanisms for their own goals, at othermembers"expense.

- The task of the mediator is to uncover and interrupt these games, and to provide an option for change. In essence, the mediator must seek out the problem and provide some type of intervention that stops the 
dysfunctional behaviours from occurring. These dysfunctional behaviours have continued and have been resistant to change. This often explains, for example, why a couple seeks the help of a mediator.

- The mediator sets forth rituals to institute change. These rituals can include a memorandum of agreement, homework sessions, seeking options from friends and relatives, seeking legal assistance, talking to a stock broker, and so on. The mediator must restate or reframe the problem so that it is more palatable to the problem seekers than their original definition of the problem. Remember: the person who defines the problem has the solution. Thus, before mediation begins, there are at least two conflicting solutions. The mediator must provide an alternative explanation for the problem so both problem seekers agree.

- The word surreptitious is often used in this context. Surreptitious essentially means covert or hidden. I use it to mean "coming through the back door." The mediator should be honest and upfront with clients, but such honesty should not preclude using methods for change which are foreign to the problem seekers.

- When the mediator positively reframes a problem, they must remember that this positive reframing is not something a mediator just does or says. Reframing should actually be something closer to a restructuring of the mediator's consciousness. Otherwise, to "learn you have to live it".

- A positive approach is always better than a negative approach. In essence, the issue of truth means less in mediation and more in other venues, such as the courts. The problem must never be allowed to be defined as "either/or." The mediator should look for what is in between, for areas of consensus, for positive results that come at the expense of neither party. Really, with respect to how the problem is defined by both parties, no one thing may be less true than the other.

- Four basic considerations for mediators with difficult clients are: the use of hypotheses, the use of circular questions and thinking, reconsidering what neutrality really means, and considering strategies.

- Hypothesizing is the assessment process. It is essentially a research technique to be used with the family. The mediator does not "search for truth." He or she searches for or attempts to put together a hypothesis of the family that makes sense of a problem.

- The hypothesis must be systemic in nature. You must be able to put all the pieces together to see the "big picture," the interlinkingrelationships.

- Hypotheses are neither true nor false: they are either useful or not useful in leading to new information about the family that later leads to positive change. Hypotheses are suppositions that generate or guide the mediator. In order for hypotheses to be useful, they must be related to the problem, but different from the client's own hypotheses about what is happening. It is your hypotheses that give order, dimension, and coherence to the pattern, flow, and direction of questions. Don't marry your own hypotheses!

- Circular questioning focuses more on the mediator than on client behaviour. It is a style, a stance, a method of questioning. Circularity is based on the idea that people are connected to each other in particular patterns through time and these patterns are identified as families. Circular questions respect 
clients and allow for fewer alliances. It is the client who is ultimately responsible for the answer. A mediator can rarely go wrong by asking questions instead of making statements. However, too many circular questions are not useful and probably would tend to confuse clients.

- A difference always reflects a relationship that is reciprocal in natureand henceforth circular.

- Linear thinking relates to cause and effect, while circular questioning techniques rely on "loop formations" that relate to issues of present and future or now-then differences.

- Use a Socratic method of questioning. The Socratic method of questioning is best practiced by posing numerous questions but providing no answers yourself. Like Socrates, the mediator knows that he or she does not know. The Socratic method of inquiry will determine the "news of a difference." Similarly, the Milan method of questioning structures the news of a relationship difference.

- The interview is the intervention. Questions by the mediator bring news of a relationship difference. A mediator should conduct the interview as a search for more information based on the feedback from the clients in response to the information the mediator receives regarding the client's relationships, and therefore about their differences that make adifference.

- Observe analogic (nonverbal) behaviours when asking questions and getting responses.

- Neutrality seems to be a misunderstood concept. Neutrality is closer to a multi positional point in time versus a non-positional reference. It refers to an attitude on the part of the mediator. At the end of the session the mediator is aligned to everyone and no one at the same time.

- Neutrality includes a sense of respect, acceptance of naïve curiosity, fascination, even admiration, for the clients and their perceivedproblem.

- The neutral mediator is not interested in blame, cause, or effect but rather in understanding how the clients are tied together in this deadlock. The mediator thus becomes a viewer of the relationship he or she haswith clients and avoids entering into the system, by trying to remain at a higher interactional level.

- Mediators promote their neutrality in the system by accepting, on one level, everything their clients state yet, on another level, rejecting it. It is the mediator's questions that allow for both to occur at once.

- A mediator's goals include met change, that is, a change in the client's ability to change. The goal is really to enhance the client's effectiveness and freedom to change.

- Mediators also have met goals, that is, they help clients to find their own self-healing capacity and discover their ownsolutions.

Strategizing is the cognitive ability to process, articulate and evaluate the effects of the past. In essence, this leads to the construction of new action plans and anticipates how to handle future difficulties. Through strategizing, mediators actively base future questions and actions (homework, consultations) on previous sessions with clients. A mediator collects and combines all information to lead to the successful management of the conflict at hand. In the "formula for success" or succession planning, the mediator must be aware of the pitfalls to the plan. Strategizing should be sensitive to the cultural, social, and legal demands of any contemplated decisions. 
This article was first published in Mediation Quarterly, (ref, 1992, 10(2), 209- 212), and is published here with kind permission of Dr Larry Fong.

Dr. Larry S Fong is a worldwide lecturer, consultant and trainer in conflict management. He has delivered mediation training to judges, police personnel, psychologists, lawyers and business persons across 14 different countries.

Dr. Fong has widely consulted with major organizations in the telecommunications, oil and gas, military, police, banking, educational and service industries.

He is a recipient of the Dr. John Haynes Memorial Award (AFMS) and the Distinguished Mediator Award (ACR). His book, „Mediation ${ }^{\text {e }}$ is published in English, German and Italian. Since 1989 his training tapes have been soldworldwide. 